\title{
Longitudinal wavevector- and frequency-dependent dielectric constant of the TIP4P water model
}

\author{
IGOR P. OMELYAN \\ Institute for Condensed Matter Physics, \\ the National Ukrainian Academy of Sciences, \\ 1 Svientsitsky St., UA-290011 Lviv, Ukraine*
}

\begin{abstract}
A computer adapted theory for self-consistent calculations of the wavevector- and frequency-dependent dielectric constant for interaction site models of polar systems is proposed. A longitudinal component of the dielectric constant is evaluated for the TIP4P water model in a very wide scale of wavenumbers and frequencies using molecular dynamics simulations. It is shown that values for the dielectric permittivity, calculated within the exact interaction site description, differ in a characteristic way from those obtained by the point dipole approximation which is usually used in computer experiment. It is also shown that the libration oscillations, existing in the shape of longitudinal time-dependent polarization fluctuations at small and intermediate wavevector values, vanish however for bigger wavenumbers. A comparison between the wavevector and frequency behaviour of the dielectric constant for the TIP4P water and the Stockmayer model is made. The static screening of external charges and damping of longitudinal electric excitations in water are considered as well. A special investigation is devoted to the time dependence of dielectric quantities in the free motion regime.
\end{abstract}

*E-mail: nep@icmp.lviv.ua 


\section{Introduction}

The study of dielectric properties of polar liquids by computer experiment is still a major challenge, given that the dielectric quantities are very sensitive to long-range intermolecular interactions and because long trajectories are required in order to obtain adequate statistical accuracy. For this reason, until now, the calculation of the wavevectorand frequency-dependent dielectric permittivity, $\varepsilon(k, \omega)$, in the whole region of $k$ and $\omega$ has been performed for the simplest model of polar systems only, namely, for the Stockmayer fluid [1]. This model is related to a class of molecular models describing interactions by point dipoles embedded in molecules. The Stockmayer system, however, does not reproduce satisfactorily the dielectric behaviour of any real polar fluid.

At the same time, more realistic interaction site (IS) models have also been considered [2-16]. In these atomic models, intermolecular potentials are presented as a sum of pairwise additive site-site terms. Although IS models do not take into account internal degrees of freedom such as electronic polarizability and, as a rule, intramolecular vibrations, they are able to reproduce experimental results in a more satisfactory way. There are two approaches to investigate the dielectric properties of IS models in both computer experiment and theory. In the first, point dipole (PD) approach, the charged sites of each molecule are replaced by a point dipole, located in the molecular centre of mass, at constructing the reaction field and the microscopic polarization vector. The site-site contributions are taken into account implicitly only, namely, in the intermolecular potentials, calculating statistical averages. In the second, IS description, the reaction and the polarization vector are constructed in view of explicit details of charge distribution within the molecule [16-18].

Main attention in early studies was directed to evaluate the static and frequencydependent dielectric constant, $\varepsilon(\omega)$, in the long-wavelength limit. In particular, such quantities have been calculated in molecular dynamics (MD) simulations for the rigid MCY [5] and TIP4P [6] models of water as well as for the flexible SPC potential [7]. The frequency dependence of the dielectric permittivity at nonzero wavevectors was investigated for the MCY [2] and TIP4P [9, 10] water models, for models of methyl cyanide

$[3,4]$, methanol [11] and methanol-water mixtures [13]. But the investigations have been restricted to small wavevector values for which the PD approach and the IS description are expected to be identical. The wavevector dependence, $\varepsilon(k)$, including high wavevector 
values, was considered in the static limit for methanol [8], methanol-water mixtures [12] and for the MCY water [14], using, however, the PD approximation. And only quite a few simulations $[15,16]$ have been performed to define the entire wavevector dependence within the exact IS description. To our knowledge, there are no computer experiment data concerning the entire frequency dependence of the dielectric permittivity of IS models at arbitrary wavenumbers.

It is necessary to point out also the following aspect of computer simulation of IS models. The calculation of the dielectric constant by MD requires explicit considerations of a finite-size medium with periodic boundaries. Usually either the cumbersome Ewald summation technique [19] or the reaction field [20] is applied for treating long ranged interactions. As is now well established for systems of point dipoles, proper calculations can be made with the both methods [21]. In the case of IS models the pattern is different. The usual point dipole reaction field (PDRF) geometry, being exact for macroscopic systems of point dipoles, may not be necessarily applicably to interpret simulation results of IS models [22]. Recently [16], it has been shown by actual calculations that uncertainties of the dielectric quantities are significant if the standard PDRF geometry is used in computer simulations. As a result, an alternative approach has been proposed. This approach deals with the IS description at constructing the reaction field and leads to the so-called interaction site reaction field (ISRF) geometry. It has been demonstrated that the ISRF geometry exhibits to be much more efficient with respect to the usual PDRF for the investigation of dielectric properties of IS models. Within the ISRF geometry, the longitudinal component, $\varepsilon_{\mathrm{L}}(k)$, of the wavevector-dependent dielectric constant for the MCY water model has been evaluated in a wide wavenumber range by Monte Carlo simulations [16]. Using correct microscopic variables for the polarization vector allows one to achieve the true infinite wavevector behaviour $\varepsilon_{\mathrm{L}}(k \rightarrow \infty)=1$.

In the present paper we propose a self-consistent theory for the calculation of the longitudinal wavevector- and frequency-dependent dielectric constant, $\varepsilon_{\mathrm{L}}(k, \omega)$, for IS models in computer experiment. This theory is applied to the TIP4P potential using MD simulations. We show that the PD approach is valid for describing the frequency dependence of the dielectric constant at very small wavenumbers only. For greater wavenumber values, the influence of higher order multipoles becomes important. Within the IS description, the longitudinal permittivity is evaluated in a rather very large scale of wavenumbers and frequencies up to the infinite wavevector and frequency limit, where $\varepsilon_{\mathrm{L}}(k, \omega) \rightarrow 1$. 


\section{The reaction field for IS models}

We consider a polar system with $N$ identical molecules composed of $M$ interaction sites which are enclosed in a volume $V$. The microscopic field, created by the molecules at point $\boldsymbol{r} \in V$ and time $t$ for a macroscopic system is equal to $\hat{\mathcal{E}}(\boldsymbol{r}, t)=\lim _{N \rightarrow \infty} \sum_{i=1}^{N} \sum_{a=1}^{M}$ $q_{a}\left\{\boldsymbol{r}-\boldsymbol{r}_{i}^{a}(t)\right\} /\left|\boldsymbol{r}-\boldsymbol{r}_{i}^{a}(t)\right|^{3}$, where $q_{a}$ and $\boldsymbol{r}_{i}^{a}(t)$ are the charge and position of site $a$ within the molecule $i$, and the sum extends over all molecules and charged sites. This sum, however, can not be calculated exactly in computer experiment which deals with finite samples. Therefore, we must restrict ourselves to a finite set of the terms for which $\left|\boldsymbol{r}-\boldsymbol{r}_{i}^{a}(t)\right| \leq R$, where $R$ is a cut-off radius. The radius $R$ does not exceed half the cell length $\sqrt[3]{V}$ if a cubic sample and the toroidal boundary conventional (tbc) are used in simulations.

Now the following problem appears. How to estimate the cut-off field caused by the summation over the unaccessible region $\left|\boldsymbol{r}-\boldsymbol{r}_{i}^{a}(t)\right|>R$ ? A solution of this problem can be found within the ISRF geometry [16]. The result for conducting boundary conditions is

$$
\hat{\mathcal{E}}(\boldsymbol{r}, t)=\sum_{i, a}^{N, M} q_{a} \Theta\left(R-\left|\boldsymbol{r}-\boldsymbol{r}_{i}^{a}(t)\right|\right)\left(\frac{\boldsymbol{r}-\boldsymbol{r}_{i}^{a}(t)}{\left|\boldsymbol{r}-\boldsymbol{r}_{i}^{a}(t)\right|^{3}}-\frac{\boldsymbol{r}-\boldsymbol{r}_{i}^{a}(t)}{R^{3}}\right)
$$

where $\Theta$ is the Heviside function, i.e., $\Theta(\rho)=1$ if $\rho \geq 0$ and $\Theta(\rho)=0$ otherwise. The first term in the right-hand side of (1) describes the usual Coulomb field, while the second contribution corresponds to the reaction field in the IS description. Performing the spatial Fourier transform of (1) one obtains

$$
\hat{\mathcal{E}}(\boldsymbol{k}, t)=\int_{V, \mathrm{tbc}} \mathrm{d} \boldsymbol{r} \mathrm{e}^{-\mathrm{i} \boldsymbol{k} \cdot \boldsymbol{r}} \hat{\mathcal{E}}(\boldsymbol{r}, t)=-4 \pi\left(1-3 \frac{j_{1}(k R)}{k R}\right) \hat{\boldsymbol{P}}_{\mathrm{L}}(\boldsymbol{k}, t)
$$

where

$$
\hat{\boldsymbol{P}}_{\mathrm{L}}(\boldsymbol{k}, t)=\frac{\mathrm{i} \boldsymbol{k}}{k^{2}} \sum_{i, a}^{N, M} q_{a} \mathrm{e}^{-\mathrm{i} \boldsymbol{k} \cdot \boldsymbol{r}_{i}^{a}(t)}
$$

is the longitudinal component for the microscopic operator of polarization density for IS models [18] and $j_{1}(z)=-\cos (z) / z+\sin (z) / z^{2}$ denotes the spherical Bessel function of first order. It is worth mentioning that as far as relativistic effects are excluded from our

consideration, the electric field (1) does not take into account retardation corrections. For the same reason, we have neglected also the contribution to the field caused by dynamical 
magnetic fields of moving charges. Because of this, the electric field has a static form and depends on time implicitly only. This field is pure longitudinal and can be defined uniquely via the longitudinal component of the polarization vector $\hat{\boldsymbol{P}}_{\mathrm{L}}(\boldsymbol{k}, t)$, that is confirmed by equation (2).

Let us apply an external electric field $\boldsymbol{E}_{0}(\boldsymbol{k}, t)$ to the system under consideration, so that the total field is $\hat{\boldsymbol{E}}(\boldsymbol{k}, t)=\boldsymbol{E}_{0}(\boldsymbol{k}, t)+\hat{\mathcal{E}}(\boldsymbol{k}, t)$. The longitudinal, wavevector- and frequency-dependent dielectric constant is defined via the material relation $4 \pi \boldsymbol{P}_{\mathrm{L}}(\boldsymbol{k}, \omega)=$ $\left(\varepsilon_{\mathrm{L}}(k, \omega)-1\right) \boldsymbol{E}_{\mathrm{L}}(\boldsymbol{k}, \omega)$, where $\boldsymbol{P}_{\mathrm{L}}(\boldsymbol{k}, \omega)=\left\langle\hat{\boldsymbol{P}}_{\mathrm{L}}(\boldsymbol{k}, \omega)\right\rangle$ and $\boldsymbol{E}_{\mathrm{L}}(\boldsymbol{k}, \omega)=\langle\hat{\boldsymbol{k}} \hat{\boldsymbol{k}} \cdot \hat{\boldsymbol{E}}(\boldsymbol{k}, \omega)\rangle$ are longitudinal components of the macroscopic polarization and total field, \langle\rangle denotes the statistical averaging at the presence of the external field, $\hat{\boldsymbol{k}}=\boldsymbol{k} / k, k=|\boldsymbol{k}|$ and the time Fourier transform $\mathscr{F}(\boldsymbol{k}, \omega)=\int_{-\infty}^{\infty} \mathrm{d} t \mathrm{e}^{-\mathrm{i} \omega t} \mathscr{F}(\boldsymbol{k}, t)$ has been used for the functions $\hat{\boldsymbol{P}}_{\mathrm{L}}(\boldsymbol{k}, t)$ and $\hat{\boldsymbol{E}}(\boldsymbol{k}, t)$. The perturbation theory of first order with respect to $\boldsymbol{E}_{0}$ yields $\left\langle\hat{\boldsymbol{P}}_{\mathrm{L}}(\boldsymbol{k}, \omega)\right\rangle=-\int_{0}^{\infty} \mathrm{d} t \mathrm{e}^{-\mathrm{i} \omega t} \frac{\mathrm{d}}{\mathrm{d} t}\left\langle\hat{\boldsymbol{P}}_{\mathrm{L}}(\boldsymbol{k}, 0) \cdot \hat{\boldsymbol{P}}_{\mathrm{L}}(-\boldsymbol{k}, t)\right\rangle_{0} \boldsymbol{E}_{0}(\boldsymbol{k}, \omega) / V k_{\mathrm{B}} T$, where \langle\rangle$_{0}$ denotes the equilibrium average at the absence of the external field, and $k_{\mathrm{B}}$ and $T$ are the Boltzmann's constant and the temperature of the system, respectively. Then, eliminating $\boldsymbol{E}_{0}(\boldsymbol{k}, \omega)$ from the presented above expressions, we obtain the desired fluctuation formula

$$
\frac{\varepsilon_{\mathrm{L}}(k, \omega)-1}{\varepsilon_{\mathrm{L}}(k, \omega)}=\frac{9 y \mathscr{L}_{\mathrm{i} \omega}\left(-\dot{G}_{\mathrm{L}}(k, t)\right)}{1+27 y \mathscr{L}_{\mathrm{i} \omega}\left(-\dot{G}_{\mathrm{L}}(k, t)\right) j_{1}(k R) /(k R)}=9 y \mathscr{L}_{\mathrm{i} \omega}\left(-\dot{g}_{\mathrm{L}}(k, t)\right) .
$$

Here

$$
G_{\mathrm{L}}(k, t)=\frac{\left\langle\hat{\boldsymbol{P}}_{\mathrm{L}}(\boldsymbol{k}, 0) \cdot \hat{\boldsymbol{P}}_{\mathrm{L}}(-\boldsymbol{k}, t)\right\rangle_{0}}{N \mu^{2}}
$$

is the longitudinal component of the wavevector-dependent dynamical Kirkwood factor for the finite sample, $\mu=\left|\boldsymbol{\mu}_{i}\right|=\left|\sum_{a}^{M} q_{a} \boldsymbol{r}_{i}^{a}\right|$ denotes the permanent magnitude of molecule's dipole moment, $\mathscr{L}_{\mathrm{i} \omega}(\ldots)=\int_{0}^{\infty} \ldots \mathrm{e}^{-\mathrm{i} \omega t} \mathrm{~d} t$ designates the Laplace transform, $y=4 \pi N \mu^{2} / 9 V k_{\mathrm{B}} T$ and $\dot{G}_{\mathrm{L}}(k, t) \equiv \partial G_{\mathrm{L}}(k, t) / \partial t$. In the case $R \rightarrow \infty$, when $j_{1}(k R) /(k R) \rightarrow 0$, the computer adapted formula (4) reduces to the fluctuation formula for macroscopic systems in terms of the infinite-system Kirkwood factor $g_{\mathrm{L}}(k, t)=$ $\lim _{R \rightarrow \infty} G_{\mathrm{L}}(k, t)$.

It is essential to emphasize that the fluctuation formula (4) takes into account finiteness of the system explicitly by the factor $j_{1}(k R) /(k R)$. As a consequence, for sufficiently large systems, the bulk $(N, V \rightarrow \infty)$ dielectric constant can be reproduced via the finite-system Kirkwood factor $G_{\mathrm{L}}(k, t)$ calculated in simulations. However, in or- 
der to achieve this self-consistency in the evaluation of the bulk dielectric constant, the equilibrium averaging in $G_{\mathrm{L}}(k, t)$ must be performed for systems with the intermolecular potential which leads exactly to the microscopic electric field $\hat{\mathcal{E}}(\boldsymbol{r}, t)$ (1) at which the fluctuation formula has been derived. This intermolecular potential can be determined via the relation $\hat{\mathcal{E}}(\boldsymbol{r}, t)=-\nabla \Phi(\boldsymbol{r}, t))$, whence $\Phi(\boldsymbol{r}, t)=\sum_{i, a}^{N, M} \phi_{i}^{a}(\boldsymbol{r}, t)$, where $\phi_{i}^{a}(\boldsymbol{r}, t)=q_{a}\left\{1 / \rho_{i}^{a}(t)+\frac{1}{2} \rho_{i}^{a}(t)^{2} / R^{3}+C\right\}, \rho_{i}^{a}(t)=\left|\boldsymbol{r}-\boldsymbol{r}_{i}^{a}(t)\right|$ and $C$ is, in general, an arbitrary constant which for infinite systems is chosen as $\left.\phi_{i}^{a}\right|_{\rho_{i}^{a} \rightarrow \infty}=0$. In our case, according to the toroidal boundary conventional, $\left.\phi_{i}^{a}\right|_{\rho_{i}^{a}=R}=0$ and, therefore, $C=-3 / 2 R^{-1}$. Then the intermolecular potential of interaction is $\sum_{a, b}^{M} q_{b} \phi_{i}^{a}\left(\boldsymbol{r}_{j}^{b}(t)\right)=\sum_{a, b}^{M} q_{a} \phi_{j}^{b}\left(\boldsymbol{r}_{i}^{a}(t)\right)$, i.e,

$$
\varphi_{i j}^{\mathrm{IS}}=\sum_{a, b}^{M} q_{a} q_{b} \Theta\left(R-\left|\boldsymbol{r}_{i}^{a}-\boldsymbol{r}_{j}^{b}\right|\right)\left(\frac{1}{\left|\boldsymbol{r}_{i}^{a}-\boldsymbol{r}_{j}^{b}\right|}+\frac{1}{2} \frac{\left|\boldsymbol{r}_{i}^{a}-\boldsymbol{r}_{j}^{b}\right|^{2}}{R^{3}}-\frac{3}{2 R}\right)
$$

where the site-site cut-off $\left|\boldsymbol{r}_{i}^{a}-\boldsymbol{r}_{j}^{b}\right| \leq R$ is performed.

The fluctuation formula (4) has already been applied previously in the long-wavelength limit at the investigation of the static and frequency-dependent dielectric constant for the MCY [5] and TIP4P [6] models. However, acting within a semiphenomenological framework, it was not understood how to perform the truncation for intermolecular potentials. As a result, the molecular cut-off $r_{i j}=\left|\boldsymbol{r}_{i}-\boldsymbol{r}_{j}\right| \leq R$, where $\boldsymbol{r}_{i}$ is the centre of mass of the $i$ th molecule, and the usual PDRF have been suggested:

$$
\varphi_{i j}^{\mathrm{PD}}=\Theta\left(R-r_{i j}\right)\left(\sum_{a, b}^{M} \frac{q_{a} q_{b}}{\left|\boldsymbol{r}_{i}^{a}-\boldsymbol{r}_{j}^{b}\right|}-\frac{\boldsymbol{\mu}_{i} \cdot \boldsymbol{\mu}_{j}}{R^{3}}\right)
$$

It is easy to show that the self-consistent potential (6) can be reduced to (7) in one case only, namely, when $d / R \rightarrow 0$, where $d=2 \max _{a}\left|\boldsymbol{r}_{i}^{a}-\boldsymbol{r}_{i}\right|$ is the diameter of the molecule. In this case, the positions for sites and the centre of mass are undistinguished within the same molecule. For IS finite samples, where $d / R \neq 0$, the PDRF potential (7) may affect on a true macroscopic behaviour of the system considerably. At the same time, the intermolecular potential $\varphi_{i j}^{\mathrm{IS}}$ corresponds completely to the conditions at which the fluctuation formula (4) has been obtained. Therefore, as far as this formula is applied to treat simulation results, the ISRF potential (6), instead of (7), must be used in computer experiment to reproduce a correct value for the dielectric constant.

Nevertheless, the molecular cut-off scheme can also be acceptable, but the PDRF potential (7) as well as the fluctuation formula (4) need to be modified. In fact, the 
intermolecular potential $\varphi_{i j}^{\mathrm{PD}}$ takes into account dipole contributions only into the reaction field. Additional terms can be identified within the molecular reaction field (MRF) approach. The result is [16]:

$$
\varphi_{i j}^{\mathrm{M}}=\Theta\left(R_{d}-r_{i j}\right)\left(\sum_{a, b}^{M} \frac{q_{a} q_{b}}{\left|\boldsymbol{r}_{i}^{a}-\boldsymbol{r}_{j}^{b}\right|}-\frac{\boldsymbol{\mu}_{i} \cdot \boldsymbol{\mu}_{j}}{R^{3}}-\frac{\mathbf{q}_{i}: \mathbf{q}_{j}-3\left(\mathbf{q}_{i}: \boldsymbol{\mu}_{j} \boldsymbol{r}_{i j}+\mathbf{q}_{j}: \boldsymbol{\mu}_{i} \boldsymbol{r}_{j i}\right)}{6 R^{5}}+\ldots\right),
$$

where $\mathbf{q}_{i}=\sum_{a}^{M} q_{a}\left(3 \boldsymbol{\delta}_{i}^{a} \boldsymbol{\delta}_{i}^{a}-\delta_{i}^{a 2} \mathbf{I}\right)$ is the quadrupole moment of the $i$ th molecule with respect to its centre of mass, $\boldsymbol{\delta}_{i}^{a}=\boldsymbol{r}_{i}^{a}-\boldsymbol{r}_{i}$, I is the unit tensor, $R_{d}=R-d / 2$, and multipoles of higher orders have been neglected. The fluctuation formula corresponding to the potential (8) can be derived as follows.

The microscopic electric field created by the molecules at point $\boldsymbol{r}$, coinciding with the centre of the spherical cavity of radius $R$, surrounded by the infinity conduction medium, can be presented in the MRF geometry as

$$
\hat{\mathcal{E}}(\boldsymbol{r}, t)=\sum_{i=1}^{N} \Theta\left(R_{d}-\left|\boldsymbol{r}-\boldsymbol{r}_{i}(t)\right|\right)\left\{\sum_{a=1}^{M} q_{a} \frac{\boldsymbol{r}-\boldsymbol{r}_{i}^{a}(t)}{\left|\boldsymbol{r}-\boldsymbol{r}_{i}^{a}(t)\right|^{3}}+\frac{\boldsymbol{\mu}_{i}(t)}{R^{3}}\right\}
$$

(quadrupole and higher-order moments do not contribute the field in the centre of the cavity). Performing the spatial Fourier transform of (9) yields after some algebra

$$
\hat{\mathcal{E}}(\boldsymbol{k}, t)=-4 \pi\left\{\sum_{\boldsymbol{k}^{\prime}} \hat{\boldsymbol{P}}\left(\boldsymbol{k}, \boldsymbol{k}^{\prime}, t\right) \frac{3 j_{1}\left(\left|\boldsymbol{k}-\boldsymbol{k}^{\prime}\right| R_{d}\right)}{\left|\boldsymbol{k}-\boldsymbol{k}^{\prime}\right| R_{d}}-\frac{j_{1}\left(k R_{d}\right)}{k R_{d}} \frac{R_{d}^{3}}{R^{3}} \hat{\boldsymbol{M}}(\boldsymbol{k}, t)\right\},
$$

where

$$
\hat{\boldsymbol{P}}\left(\boldsymbol{k}, \boldsymbol{k}^{\prime}, t\right)=\frac{\mathrm{i} \boldsymbol{k}^{\prime}}{k^{\prime 2}} \sum_{i=1}^{N} \mathrm{e}^{-\mathrm{i} \boldsymbol{k} \cdot \boldsymbol{r}_{i}(t)} \sum_{a=1}^{M} q_{a} \mathrm{e}^{-\mathrm{i} \boldsymbol{k}^{\prime} \cdot \boldsymbol{\delta}_{i}^{a}(t)}, \quad \hat{\boldsymbol{M}}(\boldsymbol{k}, t)=\sum_{i=1}^{N} \boldsymbol{\mu}_{i}(t) \mathrm{e}^{-\mathrm{i} \boldsymbol{k} \cdot \boldsymbol{r}_{i}(t)},
$$

$\boldsymbol{k}, \boldsymbol{k}^{\prime}$ are one of the allowed wavevectors $\boldsymbol{n} k_{\min }$ of the reciprocal lattice, $\boldsymbol{n}$ designates a vector with integer components and $k_{\text {min }}=2 \pi / \sqrt[3]{V}$. As we can see $\hat{\boldsymbol{P}}(\boldsymbol{k}, \boldsymbol{k}, t) \equiv \hat{\boldsymbol{P}}_{\mathrm{L}}(\boldsymbol{k}, t)$, while $\hat{\boldsymbol{M}}(\boldsymbol{k})$ is the microscopic operator of polarization density for a point dipole system. Its longitudinal component $\hat{\boldsymbol{M}}_{\mathrm{L}}(\boldsymbol{k}, t)=\hat{\boldsymbol{k}} \hat{\boldsymbol{k}} \cdot \hat{\boldsymbol{M}}(\boldsymbol{k}, t)$ coincides with $\hat{\boldsymbol{P}}_{\mathrm{L}}(\boldsymbol{k}, t)$ in the point dipole limit: $d \rightarrow 0, q_{a} \rightarrow \infty$, provided $\mu \rightarrow$ const. Using the internal field (10) and applying the perturbation theory with respect to the external field, similar to that as in the case of the ISRF geometry, we obtain the following fluctuation formula

$$
\frac{\varepsilon_{\mathrm{L}}(k, \omega)-1}{\varepsilon_{\mathrm{L}}(k, \omega)}=\frac{9 y \mathscr{L}_{\mathrm{i} \omega}\left(-\dot{G}_{\mathrm{L}}(k, t)\right)}{1-27 y\left\{\frac{\mathscr{L}_{\mathrm{i} \omega}\left(\dot{Q}_{\mathrm{L}}(k, t)\right) j_{1}\left(k R_{d}\right)}{3 k R_{d}} \frac{R_{d}^{3}}{R^{3}}-\sum_{\boldsymbol{q}(q \neq 0)} \frac{j_{1}\left(q R_{d}\right)}{q R_{d}} \mathscr{L}_{\mathrm{i} \omega}\left(\dot{G}_{\mathrm{L}}(\boldsymbol{k}, \boldsymbol{q}, t)\right)\right\}},
$$


where the summation is extended over the infinite set $\boldsymbol{n} k_{\min }$ of nonzero wavevectors $\boldsymbol{q}$,

$G_{\mathrm{L}}(\boldsymbol{k}, \boldsymbol{q}, t)=\left\langle\hat{\boldsymbol{P}}_{\mathrm{L}}(\boldsymbol{k}, \boldsymbol{k}+\boldsymbol{q}, 0) \cdot \hat{\boldsymbol{P}}_{\mathrm{L}}(-\boldsymbol{k}, t)\right\rangle_{0} / N \mu^{2}, Q_{\mathrm{L}}(k, t)=\left\langle\hat{\boldsymbol{M}}_{\mathrm{L}}(\boldsymbol{k}, 0) \cdot \hat{\boldsymbol{P}}_{\mathrm{L}}(-\boldsymbol{k}, t)\right\rangle_{0} / N \mu^{2}$ are time correlation functions and $\hat{\boldsymbol{P}}_{\mathrm{L}}\left(\boldsymbol{k}, \boldsymbol{k}^{\prime}\right)=\hat{\boldsymbol{k}} \hat{\boldsymbol{k}} \cdot \hat{\boldsymbol{P}}\left(\boldsymbol{k}, \boldsymbol{k}^{\prime}\right)$.

As was mentioned earlier, internal electric fields of classical systems are pure longitudinal. However, as it follows from the structure of equations (11), the field $\hat{\mathcal{E}}(\boldsymbol{k}, t)(10)$ for finite systems may contain a transverse component as well which vanishes in the limit $R \rightarrow \infty$ of infinite systems only. At the same time, in the ISRF geometry the field $\hat{\mathcal{E}}(\boldsymbol{k}, t)$ (2) remains by a longitudinal one even for finite systems. Therefore, from a physical point of view, the ISRF geometry is more natural than the MRF approach, because it does not influence on the true structure of electric fields. Moreover, within the MRF geometry, the fluctuation formula (12) appears to be very complicated with respect to the much more simple formula (4) in the ISRF approach. It requires the knowledge of the additional correlation functions $Q_{\mathrm{L}}(k, t)$ and $G_{\mathrm{L}}(\boldsymbol{k}, \boldsymbol{q}, t)$. While the function $Q_{\mathrm{L}}(k, t)$ can be evaluated in the usual way, the function $G_{\mathrm{L}}(\boldsymbol{k}, \boldsymbol{q}, t)$ at fixed $t$, even for spatially homogeneous systems, depends upon three parameters (magnitudes of $\boldsymbol{k}, \boldsymbol{q}$ and the cosine between them) and its calculation is a hard problem and impractical in simulations. Finally, the site-site cut-off truncation (6) has yet a minor advantage over the molecular cut-off scheme (8) because the intermolecular potential of interaction $\varphi_{i j}^{\mathrm{IS}}$ is continuous and continuously differentiable. This avoids the system energy drift associated with the passage of sites through the surface of the truncation sphere.

\section{Application to the TIP4P potential}

MD simulations have been carried out for the TIP4P potential [23] in the microcanonical ensemble at a density of $1 \mathrm{~g} / \mathrm{cm}^{3}$ and at a temperature of $T=293 \mathrm{~K}$ using the ISRF geometry (6). We considered $N=256$ molecules in the cubic volume $V=L^{3}$ to which the toroidal boundary conditions have been applied. The interaction cut-off radius was half the cell length, $R=L / 2=9.856 \AA$. The simulations were started from a well equilibrated configuration for the positions of sites, obtained by Monte Carlo simulations. Initial velocities of the molecules were generated at random with the Maxwell distribution. The equations of motion were integrated with a time step $\Delta t=2 \mathrm{fs}$ on the basis of a matrix method using the Verlet algorithm in velocity form. The system was allowed 
to achieve equilibrium for 100000 time steps. The observation time over the equilibrium state was $1000000 \Delta t=2$ ns and each 10th time step has been considered to compute the equilibrium averages. In order to provide the exact conservation for the total energy of the system during such a rather very long observation time, the velocities of atoms were slightly rescaled after every 500 time steps, so that the relative total energy fluctuations did not exceed $0.01 \%$ over the whole run. The time correlation functions were calculated with the time step $\Delta t$ in the interval of $1000 \Delta t=2 \mathrm{ps}$. The wavevector-dependent quantities were investigated for $k=[0,1, \ldots, 300] k_{\min }$, where $k_{\min }=2 \pi / L=0.319 \AA^{-1}$.

The longitudinal component $G_{\mathrm{L}}(k) \equiv G_{\mathrm{L}}(k, 0)$ of the wavevector-dependent Kirkwood factor (5) at $t=0$, obtained in the simulations within the IS description, and the corresponding function $g_{\mathrm{L}}(k) \equiv g_{\mathrm{L}}(k, 0)(4)$, related to the infinite system, are shown in fig. 1 by dashed and solid curves, respectively. In the PD approximation the diameter $d$ of the molecule is assumed to be sufficiently small in order to be entitled to replace the true microscopic operator $\hat{\boldsymbol{P}}_{\mathrm{L}}(\boldsymbol{k}, t)$ (3) of polarization density for an IS system by its analogue $\hat{\boldsymbol{M}}_{\mathrm{L}}(\boldsymbol{k}, t)=\hat{\boldsymbol{k}} \sum_{i=1}^{N} \hat{\boldsymbol{k}} \cdot \boldsymbol{\mu}_{i}(t) \mathrm{e}^{-\mathrm{i} \boldsymbol{k} \cdot \boldsymbol{r}_{i}(t)}$ for a system of point dipoles. The infinite-system Kirkwood factor calculated within the PD approximation, $\left\langle\hat{\boldsymbol{M}}_{\mathrm{L}}(\boldsymbol{k}, 0) \cdot \hat{\boldsymbol{M}}_{\mathrm{L}}(-\boldsymbol{k}, 0)\right\rangle_{0} / N \mu^{2}$, is presented in fig. 1 by open squares. For the TIP4P molecule $d=1.837 \AA$ and, therefore, the PD approximation can not be used for calculating the wavevector-dependent dielectric quantities at $k \gtrsim 2 \pi / d \sim 3.4 \AA^{-1}$. Indeed, as we can see from the figure, the PD approach reproduces values for the Kirkwood factor satisfactorily in a small region of wavenumbers only, namely, at $k<2.5 \AA^{-1}$. At greater wavevectors, these values differ considerably from those obtained within the IS description. In the infinite wavevector regime $(k \rightarrow \infty)$ the Kirkwood factor tends to $1 / 3$, when the $\mathrm{PD}$ approximation is used, whereas it approaches zero with the asymptotic behaviour $g_{\mathrm{L}}^{(\mathrm{f})}(k)$ within the IS description (see Appendix, equation (A4)). The function $g_{\mathrm{L}}^{(\mathrm{f})}(k)$ is plotted in fig. 1 by the long-short dashed curve.

In order to verify explicitly that the ISRF geometry reproduces adequately the wavevector dependence of dielectric quantities, we have performed also additional simulations for calculations of $g_{\mathrm{L}}(k)$ using the generally recognized Ewald geometry and the PDRF potential (7). These results are included in fig. 1 as well (the open circles and dotted curve). The parameters $\eta=5.76 / L$ and $k_{\max }=5 k_{\min }$ were used in the Ewald summation of Coulomb forces. As we can see from the figure, the ISRF method leads to results which are identical to those obtained within the cumbersome Ewald technique. At the 
same time, deviations of values for the wavevector-dependent Kirkwood factor obtained using the PDRF potential from those evaluated within the ISRF geometry are of order 20\%. They are well exhibited at intermediate values of wavevectors. Such a situation can be explained by the fact that the PDRF geometry does not take into account the spatial distribution of charges within the molecule at constructing the reaction field and, thus, the precision of the calculations for wavevector-dependent dielectric quantities at $k \sim 2 \pi / d$ can not exceed $d / R \sim 20 \%$. And only for great wavevector values $\left(k>6 \AA^{-1}\right)$, where the influence of boundary conditions is negligible, the both geometries become equivalent between themselves and the analytical formula (A4) can be applied here.

To analyze the wavevector-dependent dielectric function in the low frequency limit, $\omega \rightarrow 0$, it is more convenient to rewrite the fluctuation formula (4) in terms of the infinitesystem Kirkwood factor as follows

$$
\varepsilon_{\mathrm{L}}(k, \omega)=\frac{\left(1-9 y g_{\mathrm{L}}(k)\right)-9 y \mathrm{i} \omega \mathscr{L}_{\mathrm{i} \omega}\left(g_{\mathrm{L}}(k, t)\right)}{\left(1-9 y g_{\mathrm{L}}(k)\right)^{2}+(9 y)^{2} \omega^{2} \mathscr{L}_{\mathrm{i} \omega}^{2}\left(g_{\mathrm{L}}(k, t)\right)},
$$

where the equality $\mathscr{L}_{\mathrm{i} \omega}\left(-\dot{g}_{\mathrm{L}}(k, t)\right)=g_{\mathrm{L}}(k)-\mathrm{i} \omega \mathscr{L}_{\mathrm{i} \omega}\left(g_{\mathrm{L}}(k, t)\right)$ has been used. In particular, the static wavevector-dependent dielectric function $\varepsilon_{\mathrm{L}}(k)=1 /\left(1-9 y g_{\mathrm{L}}(k)\right)$ is obtained from the relation (13) putting $\omega=0$. This function, calculated within the IS description and the PD approximation, is displayed in fig. 2a as circles connected by the solid curve and as open squares, respectively. As has been pointed out previously [6], the static dielectric constant of the TIP4P water at infinite wavelengths $(k \rightarrow 0)$ appears to be smaller than the experimental value $\varepsilon_{0} \approx 80$ for real water and consists about 53. This value has been determined on the basis of MD simulations using the PDRF geometry. In our calculations within the exact ISRF geometry we have found that $\varepsilon_{0} \equiv \varepsilon_{\mathrm{L}}(k \rightarrow 0) \approx 50$. Therefore, the TIP4P water reproduces the static dielectric constant of real water even somewhat worse than this has been established earlier. The function $\varepsilon_{\mathrm{L}}(k)$ in the PD approximation behaves like that for the Stockmayer fluid [21, 24]. For example, in the infinite wavevector limit, the dielectric constant tends to the wrong Onsager limit value $1 /(1-3 y)=-0.0649$.

The true wavevector behaviour for the dielectric constant can be reproduced within the IS description only. As we can see, the static dielectric constant of the TIP4P water is negative over a wavevector range bounded by two singularities, where $9 y g_{\mathrm{L}}(k) \rightarrow 1$ and $\varepsilon_{\mathrm{L}}(k) \rightarrow \pm \infty$. The first singularity is achieved at $k=k_{\mathrm{I}} \approx 0.297 \AA^{-1}$, whereas the second 
singularity may be estimated [14] via the relation

$$
k_{\mathrm{II}} \approx \sqrt{\frac{9 y}{\mu^{2}} \sum_{a=1}^{M} q_{a}^{2}}=\sqrt{\frac{4 \pi N}{V k_{\mathrm{B}} T} \sum_{a=1}^{M} q_{a}^{2}} .
$$

This relation is derived solving the equation $9 y g_{\mathrm{L}}^{(\mathrm{f})}(k)=1$ and neglecting intersite contributions (the second term in the right-hand side of $(\mathrm{A} 4))$ in the intramolecular part $g_{\mathrm{L}}^{(\mathrm{f})}(k)$ of the Kirkwood factor. Applying the relation (14) yields for the TIP4P molecule $k_{\mathrm{II}} \approx$ $19.71 \AA^{-1}$, that is in an excellent accord with the simulation result $20.28 \AA^{-1}$. At $k>k_{\text {II }}$ the function $\varepsilon_{\mathrm{L}}(k)$, remaining positive, slightly approaches unity at $k \gtrsim 100 \AA^{-1}$. In the low frequency limit at $k^{\star}=k_{\mathrm{I}}, k_{\mathrm{II}}$ we put $1-9 y g_{\mathrm{L}}\left(k^{\star}\right)=0$ and obtain from (13) that the dielectric constant behaves as $\varepsilon_{\mathrm{L}}\left(k^{\star}, \omega\right)=-\mathrm{i} / \omega \tau_{\mathrm{L}}^{\text {cor }}\left(k^{\star}\right)$, where $\tau_{\mathrm{L}}^{\text {cor }}(k)=\int_{0}^{\infty} g_{\mathrm{L}}(k, t) / g_{\mathrm{L}}(k) \mathrm{d} t$ is the correlation time. Therefore, the real part of the dielectric permittivity in this regime is equal to zero, whereas the imaginary part tends to infinity as $1 / \omega$. This result can be better understood introducing the generalized coefficient $\sigma_{\mathrm{L}}(k, \omega)$ of polarization conductivity, which defines the macroscopic current $\boldsymbol{I}_{\mathrm{L}}(\boldsymbol{k}, t)=\partial \boldsymbol{P}_{\mathrm{L}}(\boldsymbol{k}, t) / \partial t$ in the frequency representation as $\boldsymbol{I}_{\mathrm{L}}(\boldsymbol{k}, \omega)=\sigma_{\mathrm{L}}(k, \omega) \boldsymbol{E}_{\mathrm{L}}(\boldsymbol{k}, \omega)$. Taking into account that in this representation $\boldsymbol{I}_{\mathrm{L}}(\boldsymbol{k}, \omega)=\mathrm{i} \omega \boldsymbol{P}_{\mathrm{L}}(\boldsymbol{k}, \omega)$, we can express the polarization conductivity in terms of the dielectric constant as $\sigma_{\mathrm{L}}(k, \omega)=\frac{\mathrm{i} \omega}{4 \pi}\left(\varepsilon_{\mathrm{L}}(k, \omega)-1\right)$. Thus in the limit of small frequencies we have that $\sigma_{\mathrm{L}}(k)=\lim _{\omega \rightarrow 0} \sigma_{\mathrm{L}}(k, \omega)=1 / 4 \pi \tau_{\mathrm{L}}^{\text {cor }}(k) \neq 0$ if $k \in k^{\star}$ and $\sigma_{\mathrm{L}}(k)=0$ otherwise.

In view of the existence of a nonvanishing coefficient for the generalized conductivity in the static limit, the following question arises. Does it violate the well-known law that static macroscopic currents are absent in the dielectrics? We can be sure that this law remains in force, because $\sigma_{\mathrm{L}}(k) \neq 0$ when $\left|\varepsilon_{\mathrm{L}}(k)\right| \rightarrow \infty$ and, therefore, the total electric field in the system vanishes because of $\boldsymbol{E}_{\mathrm{L}}(\boldsymbol{k})=\hat{\boldsymbol{k}} \hat{\boldsymbol{k}} \cdot \boldsymbol{E}_{0}(\boldsymbol{k}) / \varepsilon_{\mathrm{L}}(k) \rightarrow 0$, so that the polarization current does not appear. In this case, the longitudinal external field is compensated completely by the internal field of polar molecules. Moreover, the singularities in the dielectric permittivity do not lead to singularities in physically observing quantities. This is so because these quantities are expressed through the external electric field using multipliers of type $1 / \varepsilon_{\mathrm{L}}(k)$ or $\left(\varepsilon_{\mathrm{L}}(k)-1\right) / \varepsilon_{\mathrm{L}}(k)$ which are free of singularities. For instance the ratio $\mathcal{E}(\boldsymbol{k}) /\left(\hat{\boldsymbol{k}} \hat{\boldsymbol{k}} \cdot \boldsymbol{E}_{0}(\boldsymbol{k})\right)$ of the macroscopic static field of polar molecules and the external field is determined by the factor $-\left(\varepsilon_{\mathrm{L}}(k)-1\right) / \varepsilon_{\mathrm{L}}(k)=-9 y g_{\mathrm{L}}(k)$. The sign minus shows that the field of molecules is opposite to the external field, because molecular dipole moments align always along external fields. The maximum magnitude of this factor ranges 
up to about 44.9 at $k \approx 3.03 \AA^{-1}$ (the first maximum of the Kirkwood factor), where the dielectric constant takes its almost maximum value -0.0228 within the negative region. The value $3.03 \AA^{-1}$ corresponds to the wavelength $\lambda=2 \pi / k \approx 2.07 \AA \sim d$. Therefore, optimal conditions (maximal average torques acting on molecules due to electric fields) for alignment of dipoles along external fields are observed when spatial inhomogeneity of these fields has a characteristic length of its varying in coordinate space of order of the molecular diameter (the so called spatial resonance). A similar wavevector behaviour of $\varepsilon_{\mathrm{L}}$ is exhibited for a central force model of water [15].

The knowledge of the dielectric constant in the whole region of wavenumbers allows one to solve the problem of static screening of external charges in water. An external charge, enclosed in the dielectric, causes a polarization of the system. The potential of the electric field, created in such a way, can be written in the $\boldsymbol{k}$-representation as $\varphi(\boldsymbol{k})=$ $\varphi^{(0)}(\boldsymbol{k}) / \varepsilon_{\mathrm{L}}(k)$, where $\varphi^{(0)}(\boldsymbol{k})=4 \pi / k^{2}$ is the Fourier transform for the potential $\varphi^{(0)}(\boldsymbol{r})=$ $1 / r$ of an unit charge in the vacuum. Then applying the inverse Fourier transform to the function $\varphi(\boldsymbol{k})$ one obtains $\varphi(\boldsymbol{r})=\frac{1}{(2 \pi)^{3}} \int \varphi(\boldsymbol{k}) \mathrm{e}^{\mathrm{i} \boldsymbol{k} \cdot \boldsymbol{r}} \mathrm{d} \boldsymbol{k}=\frac{2}{\pi} \int_{0}^{\infty} \frac{1}{\varepsilon_{\mathrm{L}}(k)} \frac{\sin k r}{k r} \mathrm{~d} k=\varphi^{(0)}(\boldsymbol{r}) / \varepsilon_{\mathrm{L}}(r)$. The function $1 / \varepsilon_{\mathrm{L}}(r)$, which is plotted in fig. $2 \mathrm{~b}$, describes the static screening. It can be shown easily, using the asymptotic behaviour (A4), that the dielectric constant in the infinite wavenumber regime is expanded over inverse wavevectors as $\varepsilon_{\mathrm{L}}(k)=1+1 /\left(r_{\mathrm{D}} k\right)^{2}+$ $\mathcal{O}\left(k^{-4}\right)$, where $r_{\mathrm{D}}=1 / k_{\mathrm{II}}$. The expression $\varepsilon_{\mathrm{L}}(k)=1+1 /\left(r_{\mathrm{D}} k\right)^{2}$ is often used in the whole range of wavevectors, considering processes of static screening in plasma. This leads to the well-known Debye exponential screening $1 / \varepsilon_{\mathrm{L}}(r)=\exp \left(-r / r_{\mathrm{D}}\right)$ with the Debye's radius $r_{\mathrm{D}}$. In the case of dielectrics the pattern is different. The function $1 / \varepsilon_{\mathrm{L}}(r)$, starting from 1 at $r=0$, exhibits a pronounced oscillatory feature, reflecting the influence of the microscopic structure of the system. And only beginning from distances to the external charge of order $r \gtrsim 15 \AA$ this function tends to its value $1 / \varepsilon_{0}$ in the macroscopic limit.

Examples for the normalized autocorrelation functions $\Phi_{\mathrm{L}}(k, t)=G_{\mathrm{L}}(k, t) / G_{\mathrm{L}}(k)(5)$ and $\phi_{\mathrm{L}}(k, t)=g_{\mathrm{L}}(k, t) / g_{\mathrm{L}}(k)(4)$, describing the time decay of longitudinal polarization fluctuations in the finite $(N=256)$ and infinite systems are plotted in fig. 3 by the circles and solid curves, respectively (the infinite-system functions are obtained applying the inverse Laplace transform to the relation (4)). The curves are for a set of wavevectors accessible in the simulations. For the purpose of comparison, analogous functions, $\left\langle\hat{\boldsymbol{M}}_{\mathrm{L}}(\boldsymbol{k}, 0) \cdot \hat{\boldsymbol{M}}_{\mathrm{L}}(-\boldsymbol{k}, t)\right\rangle_{0} /\left\langle\hat{\boldsymbol{M}}_{\mathrm{L}}(\boldsymbol{k}, 0) \cdot \hat{\boldsymbol{M}}_{\mathrm{L}}(-\boldsymbol{k}, 0)\right\rangle_{0}$, obtained within the PD approximation for the finite system, are also presented in this figure by dashed curves. The self 
$(i=j$ in equation $(\mathrm{A} 1))$ part $\phi_{\mathrm{L}}^{(\mathrm{s})}(k, t)=g_{\mathrm{L}}^{(\mathrm{s})}(k, t) / g_{\mathrm{L}}^{(\mathrm{s})}(k)$ of $\phi_{\mathrm{L}}(k, t)$ and the normalized function $\phi_{\mathrm{L}}^{(\mathrm{f})}(k, t)=g_{\mathrm{L}}^{(\mathrm{f})}(k, t) / g_{\mathrm{L}}^{(\mathrm{f})}(k)$ (note that $g_{\mathrm{L}}^{(\mathrm{s})}(k)=g_{\mathrm{L}}^{(\mathrm{f})}(k)$ in the static limit) corresponding to a noninteracting system, calculated with the help of stochastic simulations according to equation (A2), are included in fig. 3 as well and shown by the dotted and long-short dashed curves, respectively. As we can see, the self portion of $g_{\mathrm{L}}(k, t)$ begins to dominate already at $k \geq 10 k_{\min }$ and the free motion regime starts at $k \geq 40 k_{\min }$. The normalized function $g_{\mathrm{L}}^{(\mathrm{f})}(k, t)$, evaluated via the analytical formula (A3), is plotted in insets (e)-(h) of fig. 3 by open circles.

The time correlation functions obtained in the IS description are identical to those within the PD approximation in the zero wavevector limit only but they differ from one another significantly at greater $\left(k>k_{\min }\right)$ wavevector values. For example, in the infinite wavevector limit (see fig. $3 g, h$ ), the PD functions exhibit the pure Gaussian behaviour $f_{\mathrm{G}}(k, t)$, whereas the IS functions have a more complicated structure of the time dependence (A3). Taking into account the remarks concerning the behaviour of these functions in the static limit (see fig. 1), we conclude that the PD approximation, being exact for systems of point dipoles, is unsuitable, in general, to investigate the wavevector- and frequency-dependent dielectric constant of IS models of polar fluids.

The most striking feature in the time behaviour for the longitudinal component of the infinite-system function $\phi_{\mathrm{L}}(k, t)$ is damped oscillations superimposed on the exponential. They are called librational oscillations and can be observed for the self part $\phi_{\mathrm{L}}^{(\mathrm{s})}(k, t)$ as well. To show these oscillations in more detail, we have also presented 3D plot of the function $\phi_{\mathrm{L}}(k, t)$ in fig. 4. The librational oscillations appear for systems with a slowly relaxing character of intermolecular torques and describe the rapid motion of a molecule in the average electric field $\sim \frac{r}{r^{3} \varepsilon_{\mathrm{L}}^{(r)}}$ of its neighbours (see fig. $2 \mathrm{~b}$ ). It is not a surprise, the librations are absent in the shape of the free-motion functions $\phi_{\mathrm{L}}^{(\mathrm{f})}(k, t)$ which can be related to polarization fluctuations of a dilute polar fluid. The librational oscillations have been found previously for the MCY $[2,5]$ and TIP4P potentials [6] and for a model of methyl cyanide [3]. However, the investigation of the librational oscillations was restricted to zero and very small values $\left(\sim k_{\text {min }}\right)$ of wavevector. In our study, performed in a rather very large scale of wavenumbers, we have identified the librational oscillations at small and intermediate wavenumber values, namely, at $k \lesssim 10 k_{\min } \sim 3 \AA^{-1}$ (see figs. 3, 4). They vanish at bigger wavevectors when the time correlation functions behave like those for a noninteracting system. 
It is necessary to underline that all the functions after a sufficiently long period begin to decay purely exponentially in time as $\sim \exp \left(-t / \tau_{\mathrm{L}}^{\mathrm{rel}}(k)\right)$, where $\tau_{\mathrm{L}}^{\mathrm{rel}}(k)$ is the relaxation time. The wavevector-dependent relaxation $\tau_{\mathrm{L}}^{\text {rel }}(k)$ as well as the correlation $\tau_{\mathrm{L}}^{\text {cor }}(k)$ times for the finite and infinite systems are shown in fig. 5. At $k=0$ we have found for the finite-system function $\tau^{\mathrm{rel}}(0)=6.7 \mathrm{ps}$ and $\tau^{\mathrm{cor}}(0)=6.4 \mathrm{ps}$. As was pointed out above, the correlation functions have been evaluated in the finite time interval $t \in[0,2 \mathrm{ps}]$. The relaxation times will be used by us to calculate contributions at time integration for $t>2 \mathrm{ps}$, where all the correlation functions exhibit almost purely relaxation behaviour. We indicate the longest tails in the time correlation functions for the infinite system at $k \sim 1.7$ and $2.9 \AA^{-1}$. These functions remain positive anywhere in time space, contrary to the Stockmayer fluid with the dipolaron behaviour $[1,25]$ of dipole moment fluctuations.

Now, we are in a position to discuss the result of calculations (4) for the longitudinal wavevector- and frequency-dependent dielectric constant $\varepsilon_{\mathrm{L}}(k, \omega)$ of the TIP4P water. Real $\varepsilon_{\mathrm{L}}^{\prime}(k, \omega)$ and imaginary $\varepsilon_{\mathrm{L}}^{\prime \prime}(k, \omega)$ parts of $\varepsilon_{\mathrm{L}}(k, \omega)=\varepsilon_{\mathrm{L}}^{\prime}(k, \omega)-\mathrm{i} \varepsilon_{\mathrm{L}}^{\prime \prime}(k, \omega)$ are shown in figs. 6 and 7 as solid and dashed curves, respectively. The calculation of the frequencydependent dielectric constant for the TIP4P water has already been dealt with in the paper [6]. In this paper, however, the dielectric relaxation was investigated at zero wavevector value only. Moreover, the simulation results here were actually deduced within the PDRF potential rather than the exact ISRF geometry. As has been concluded previously [16], investigating the wavevector dependence of the dielectric constant of the MCY model, and shown above for the wavevector-dependent Kirkwood factor $g_{\mathrm{L}}(k)$ of the TIP4P water (see fig.1), it is risky to use the PDRF geometry for systems of hundreds molecules. Although it is hard to tell except by numerical computations whether the results [6] for the frequency dependence of the dielectric constant differ significantly from the exact results corresponding to the macroscopic system. For this reason we have repeated the calculation of the frequency-dependent dielectric constant $\varepsilon(\omega)=\lim _{k \rightarrow 0} \varepsilon_{\mathrm{L}}(k, \omega)$. The result of these calculations, performed in the ISRF geometry, is shown in fig. 6a. Comparing the previous result (cf. fig. 6 of ref. 6) with our one, we can observe that apart from the slight differences in the low frequency regime the agreement is quite good for higher frequencies. Therefore, the dielectric constant is less sensitive to boundary effects at intermediate and high values of frequency.

Since all the correlation functions decay exponentially at great times, in the low frequency regime the dielectric permittivity behaves like the Debye dielectric for arbitrary 
wavevectors. Deviations can be visible at higher frequencies and at small and intermediate wavevector values, where the collective molecular librations take an important role in forming the polarization fluctuations. Above some $\omega=1-10 \mathrm{THz}$, where $\mathrm{THz}=\mathrm{ps}^{-1}$, the relaxation process gradually changes into a resonance process characterized by an frequency of 60-200 THz (which depends on wavevector) and reflecting the rapid librational motion of the molecules. The main differences at zero wavevector between the frequencydependent dielectric constant for the TIP4P water, Debye and Stockmayer models as well as real water have been done already $[6,26]$. Now we consider differences in the behaviour on frequency between the longitudinal dielectric constant of the TIP4P water and the Stockmayer model [1] at nonzero wavevector values.

Despite a similar overall shape of $\varepsilon_{\mathrm{L}}(k, \omega)$ to that of the Stockmayer fluid, additional features emerge for the TIP4P water, namely, a second maximum in the imaginary part $\varepsilon_{\mathrm{L}}^{\prime \prime}(k, \omega)$ at $k<7 k_{\min }$ and a more complicated structure for the real part $\varepsilon_{\mathrm{L}}^{\prime}(k, \omega)$ at $k<20 k_{\text {min }}$. For example, the real part $\varepsilon_{\mathrm{L}}^{\prime}(k, \omega)$ for the Stockmayer system always increases at increasing frequency, while we can observe clearly additional maximum and minimum for the TIP4P water in the librational resonance range. Although the TIP4P model does not reproduce the dielectric properties of water at all well, since it does not account for polarizability and the coupling of inter- and intramolecular motions, a similar structure in the dielectric constant of real water for nonzero wavevector values may be present as well, because in real water the librational frequency is well separated from the lowest intramolecular vibration [27]. It is interesting also to point out that the dielectric constant of the TIP4P water at frequencies $\omega>10 \mathrm{THz}$ practically does not depend on wavevector in the range $0 \leq k \leq 2 k_{\min }$ and even up to $k=10 k_{\min }$ at $\omega>200 \mathrm{THz}$ and remains the same as in the low wavevector limit (cf. fig. 6b-e). The wavevector- and frequencydependent dielectric constant at great wavenumber values is shown in fig. 7. As we can see, the correct value of nonpolarizable systems in the infinite wavevector and frequency limit, $\varepsilon_{\infty}=1$, can only be obtained if the dielectric constant is known up to about $k \sim 150 k_{\min } \approx 50 \AA^{-1}$ and $\omega \sim 1000 \mathrm{THz}$. It is worth to remark also that at $k \geq 20 k_{\min }$ the frequency dependence of the dielectric constant can be reproduced quantitatively using the analytical formula (A3) for the time correlation functions of a noninteracting system. The dielectric constant, calculated in such a way, is shown in fig. $6 \mathrm{f}-\mathrm{h}$ and fig. 7 by circles.

The dielectric constant $\varepsilon_{\mathrm{L}}(k, \omega)$ at fixed real values of wavevector and frequency de- 
scribes the response of the system on longitudinal electric fields in the form of monochromatic plane waves, i.e., when $E(\boldsymbol{r}, t) \sim \mathrm{e}^{\mathrm{i}(\omega t-\boldsymbol{k} \cdot \boldsymbol{r})}$. Arbitrary inhomogeneous fields can be presented by a set of the plane waves via the time and spatial Fourier transform. Therefore, the response on such fields can be determined integrating the corresponding monochromatic contributions over the frequency and wavenumber spectrum using $\varepsilon_{\mathrm{L}}(k, \omega)$. The necessity of introducing pure imaginary frequencies $\omega \equiv \mathrm{i} \omega^{*}$ with $\omega^{*}>0$ arises in the problem of finding electric fields $E(\boldsymbol{r}, t) \sim \mathrm{e}^{\left(-\omega^{*} t-\mathrm{i} \boldsymbol{k} \cdot \boldsymbol{r}\right)}$ in the system when the external field is turned off. Such damping in time electric fields can be obtained using the dispersion relation $\varepsilon_{\mathrm{L}}(k, \omega)=0$ [28]. It can be seen from figs. 6,7 that this relation is not satisfied at real frequencies. So that, contrary to transverse electromagnetic waves, longitudinal monochromatic waves can not propagate without external sources of charges. From the fluctuation formula (4) it follows that $\varepsilon_{\mathrm{L}}(k, \omega) \rightarrow 0$ when $\mathscr{L}_{\mathrm{i} \omega}\left(-\dot{g}_{\mathrm{L}}(k, t)\right) \rightarrow \infty$. The last limit can be achieved at imaginary frequencies $\omega=\mathrm{i} \omega^{*}$ only, where $\omega^{*}(k) \rightarrow 1 / \tau_{\mathrm{L}}^{\mathrm{rel}}(k)$. Thus, the electric fields are damped exponentially with the characteristic interval of order of the relaxation time. Maximal life times of the longitudinal electric excitations are observed at spatial inhomogeneity of $k \sim 1.7 \AA^{-1}$ (see fig. 5) and consists about 1.6ps. Strong spatially inhomogeneous fields $(k \rightarrow \infty)$ disappear immediately after disappearing external supporting fields.

Finally, we consider some aspects of applying the well-known Kramers-Kronig relations to the longitudinal wavevector- and frequency-dependent dielectric constant. In general, these relations connect real $\xi^{\prime}(\omega)$ and imaginary $\xi^{\prime \prime}(\omega)$ parts of the function $\xi(\omega)=\xi^{\prime}(\omega)-$ $\mathrm{i} \xi^{\prime \prime}(\omega)$ of frequency as

$$
\xi^{\prime}(\omega)=\frac{2}{\pi} \int_{0}^{\infty} \frac{x \xi^{\prime \prime}(x)}{x^{2}-\omega^{2}} \mathrm{~d} x, \quad \xi^{\prime \prime}(\omega)=-\frac{2 \omega}{\pi} \int_{0}^{\infty} \frac{\xi^{\prime}(x)}{x^{2}-\omega^{2}} \mathrm{~d} x
$$

From the mathematical point of view the relations (15) are fulfilled if the function $\xi(\omega)$ can be presented in the form $\mathscr{L}_{\mathrm{i} \omega}(\xi(t))$, where $\xi(t)$ is a real analytical function of time. Then, taking into account the fluctuation formulas (4), it can be shown that in two cases at least, namely, when $\xi(\omega) \equiv \varepsilon_{\mathrm{L}}(0, \omega)-1=\varepsilon(\omega)-1$ and $\xi(\omega) \equiv\left(\varepsilon_{\mathrm{L}}(k, \omega)-1\right) / \varepsilon_{\mathrm{L}}(k, \omega)$ for arbitrary $k$, the Kramers-Kronig relations will be satisfied. At the same time it is not obvious that these relations must be valid for the function $\xi(\omega) \equiv \varepsilon_{\mathrm{L}}(k, \omega)-1$ at $k \neq 0$. We have established on the basis of our numerical analysis that the Kramers-Kronig relations can apply to the longitudinal dielectric constant in the form $\varepsilon_{\mathrm{L}}(k, \omega)-1$ at 
such wavenumbers only, where the static dielectric constant $\varepsilon_{\mathrm{L}}(k)$ takes positive values, i.e., when $0 \leq k<k_{\mathrm{I}}$ and $k>k_{\mathrm{II}}$. Physically, the impossibility to use the function $\varepsilon_{\mathrm{L}}(k, \omega)-1$ for the transformations (15) at arbitrary wavenumbers lies in the fact that, in fact, the external field $\boldsymbol{E}_{0}$, but not the total field $\boldsymbol{E}$, can be considered as an independent parameter which causes time varying all observing quantities in the system.

\section{Conclusion}

A fluctuation formula suitable for the self-consistent calculations of the wavevectorand frequency-dependent dielectric constant for interaction site models of polar systems has been rigorously derived in the situation that is typical for computer simulations. Using this formula, the longitudinal component of the dielectric constant has been evaluated by the MD method for the TIP4P model of water in a very wide scale of wavenumbers and frequencies. The most striking feature of interaction site models consists in existing of the libration oscillations in the shape of longitudinal time-dependent polarization fluctuations and this feature is reproduced by the TIP4P potential as well. We have showed, however, that the libration oscillations vanish at increasing wavenumber. Choosing the correct microscopic variable for polarization density, which corresponds completely to interaction site models, allows us to investigate the frequency dependence of the dielectric constant for arbitrary wavevector values. At the same time, it has been corroborated by the explicit calculations that the PD approximation is unsuitable, in general, for evaluating the wavevector- and frequency-dependent dielectric constant of interaction site models of polar fluids.

Since it has now been shown that the calculation of the wavevector- and frequencydependent dielectric constant in computer simulations for a given interaction site model is practical in principle, we believe that this fact will stimulate further research in both theory and pure experiment. A next paper of this series will be devoted to a more complicated case, namely, to the calculation of the transverse wavevector- and frequencydependent dielectric constant for the TIP4P water.

The author would like to acknowledge financial support of the President of Ukraine. 


\section{References}

[1] Omelyan, I.P., 1996, Molec. Phys., 87, 1273.

[2] Impey, R.W., Madden, P.A., And McDonald, I.R., 1982, Molec. Phys., 46, 513.

[3] Edwards, D.M.F., Madden, P.A., And McDonald, I.R., 1984, Molec. Phys., 51, 1141.

[4] Edwards, D.M.F., And Madden P.A., 1984, Molec. Phys., 51, 1163.

[5] Neumann, M., 1985, J. Chem. Phys., 82, 5663.

[6] Neumann, M., 1986, J. Chem. Phys., 85, 1567.

[7] Anderson, J., Ullo, J.J., And Yip, S., 1987, J. Chem. Phys., 87, 1726.

[8] Fonseca, T., and Ladanyi, B.M., 1990, J. Chem. Phys., 93, 8148.

[9] Bertolini, D., And Tani, A., 1992, Molec. Phys., 75, 1047.

[10] Bertolini, D., And Tani, A., 1992, Molec. Phys., 75, 1065.

[11] Skaf M.S., Fonseca T., and Ladanyi B.M., 1993, J. Chem. Phys., 98, 8929.

[12] Skaf M.S., and Ladanyi B.M., 1995, J. Chem. Phys., 102, 6542.

[13] Ladanyi B.M., and Skaf M.S., 1996, J. Phys. Chem., 100, 1368.

[14] Omelyan, I.P., 1996, Phys. Lett. A, 220, 167.

[15] Bopp, P.A., Kornyshev A.A., and Sutmann G., 1996, Phys. Rev. Lett., 76, 1280.

[16] Omelyan, I.P., 1996, Phys. Lett. A, 223, 295.

[17] Raineri, F.O., Zhou, Y., Friedman, H.L., and Stell, G., 1991, Chem. Phys., 152, 201.

[18] Raineri, F.O., Resat, H., and Friedman, H.L., 1992, J. Chem. Phys., 96, 3068.

[19] Allen, M.P., And Tildesley, D.J., 1987, Computer Simulation of Liquids (Oxford: Clarendon).

[20] Barker, J.A., And Watts, R.O., 1973, Molec. Phys., 26, 789.

[21] Neumann, M., 1986, Molec. Phys., 57, 97.

[22] Friedman, H.L., 1975, Molec. Phys., 29, 1533.

[23] Jorgensen, W.L., Chandrasekhar, J., Madura, J.D., Impey, R.W. And KleIN, M.L., 1983, J. Chem. Phys., 79, 926.

[24] Omelyan, I.P., 1995, Phys. Lett. A, 208, 237.

[25] Pollock, E.L., And Alder, B.J., 1981, Phys. Rev. Lett., 46, 950.

[26] Neumann, M., Steinhauser, O., and Pawley, G.S., 1984, Molec. Phys., 52, 97.

[27] Hasted, J.B., 1973, Aqueous Dielectrics (London: Chapman and Hall).

[28] Bredov, M.M., Rumyantsev V.V., And Toptigin I.N., 1985, Classical Electrodynamics (Moscow: Nauka). 


\section{Appendix}

We now consider the time dependence of the longitudinal wavevector-dependent Kirkwood factor

$$
g_{\mathrm{L}}(k, t)=\frac{1}{N \mu^{2} k^{2}}\left\langle\sum_{i, j ; a, b}^{N ; M} q_{a} q_{b} \mathrm{e}^{\mathrm{i} \boldsymbol{k} \cdot\left(\boldsymbol{r}_{i}^{a}(t)-\boldsymbol{r}_{j}^{b}(0)\right)}\right\rangle_{0}
$$

in the free motion regime. In this case, molecules are statistically independent of one another and, therefore, nonzero contributions to $g_{\mathrm{L}}(k, t)$ give only the self (intramolecular) part of terms with coincident molecular indexes $(i=j)$ of summation (A1). The site velocities of molecules can be presented as $\boldsymbol{v}_{i}^{a}(t)=\boldsymbol{V}_{i}+\boldsymbol{\Omega}_{i}(t) \times \boldsymbol{\delta}_{i}^{a}(t)$, where $\boldsymbol{V}_{i}$ and $\boldsymbol{\Omega}_{i}$ are the translational and angular velocities of the $i$ th molecule, respectively. We note that according to the Euler equations, the angular velocities depend on time even for free rotational motion, so that $\boldsymbol{r}_{i}^{a}(t)=\boldsymbol{r}_{i}(0)+\boldsymbol{V}_{i} t+\boldsymbol{\delta}_{i}^{a}(t)$, where $\boldsymbol{\delta}_{i}^{a}(t)=\boldsymbol{\delta}_{i}^{a}(0)+\int_{0}^{t}\left[\boldsymbol{\Omega}_{i}\left(t^{\prime}\right) \times \boldsymbol{\delta}_{i}^{a}\left(t^{\prime}\right)\right] \mathrm{d} t^{\prime}$. Then, taking into account that the translational and angular velocities of each molecule are distributed independently at equilibrium, the expression (A1) transforms into

$$
g_{\mathrm{L}}^{(\mathrm{f})}(k, t)=\frac{1}{N \mu^{2} k^{2}} \sum_{i=1}^{N}\left\langle\mathrm{e}^{\mathrm{i} \boldsymbol{k} \cdot \boldsymbol{V}_{i} t}\right\rangle_{0}\left\langle\sum_{a, b}^{M} q_{a} q_{b} \mathrm{e}^{\mathrm{i} \boldsymbol{k} \cdot\left(\boldsymbol{\delta}_{i}^{a}(t)-\boldsymbol{\delta}_{i}^{b}(0)\right)}\right\rangle_{0} .
$$

The first averaging in (A2) can easily be evaluated applying the Maxwell distribution $f_{0}\left(V_{\alpha}\right)=\left(\frac{m}{2 \pi k_{\mathrm{B}} T}\right)^{1 / 2} \exp \left(-\frac{m V_{\alpha}^{2}}{2 k_{\mathrm{B}} T}\right)$ with respect to the cartesian components $(\alpha=x, y, z)$ of the translational velocity $\boldsymbol{V}$, where $m$ is the mass of the molecule. As a result, we obtain $\left\langle\mathrm{e}^{\mathrm{i} \boldsymbol{k} \cdot \boldsymbol{V} t}\right\rangle_{0}=$ $\iiint_{-\infty}^{\infty} \mathrm{d} V_{x} \mathrm{~d} V_{y} \mathrm{~d} V_{z} f_{0}\left(V_{x}\right) f_{0}\left(V_{y}\right) f_{0}\left(V_{z}\right) \exp \left(\mathrm{i}\left(k_{x} V_{x}+k_{y} V_{y}+k_{z} V_{z}\right)\right)=\exp \left(-\frac{k_{\mathrm{B}} T}{2 m} k^{2} t^{2}\right)=f_{\mathrm{G}}(k, t)$. The second averaging in (A2) is not reduced, in general, to an analytic form. However, due to the fact that the Gaussian multiplier $f_{\mathrm{G}}(k, t)$ quickly decays to zero with increasing $t$ at $k \neq 0$, we can cast the solution for $\boldsymbol{\delta}_{i}^{a}(t)$ into the Taylor series by writing $\boldsymbol{\delta}_{i}^{a}(t)=\boldsymbol{\delta}_{i}^{a}+\left[\boldsymbol{\Omega}_{i} \times \boldsymbol{\delta}_{i}^{a}\right] t+\mathcal{O}\left(t^{2}\right)$, where $\boldsymbol{\Omega}_{i}$ and $\boldsymbol{\delta}_{i}^{a}$ are taken at time $t=0$ and terms of order $t^{2}$ and higher have been neglected. The integration over angular velocities it is convenient to perform in the moving coordinate system $X Y Z$, attached to the molecule, in which the Maxwell distribution has the form $f_{0}\left(\Omega_{\alpha}\right)=\left(\frac{J_{\alpha}}{2 \pi k_{\mathrm{B}} T}\right)^{1 / 2} \exp \left(-\frac{J_{\alpha} \Omega_{\alpha}^{2}}{2 k_{\mathrm{B}} T}\right)$, where $J_{\alpha}$ denote the moments of inertia along principal axes $\alpha=X, Y, Z$. Finally, performing averaging over orientations of the molecule with respect to the laboratory frame $x y z$, we obtain the desired result

$$
\begin{aligned}
g_{\mathrm{L}}^{(\mathrm{f})}(k, t) & =\frac{f_{\mathrm{G}}(k, t)}{4 \pi \mu^{2} k^{2}} \sum_{a, b}^{M} q_{a} q_{b} \int_{0}^{\pi} \sin \theta \mathrm{d} \theta \int_{0}^{2 \pi} \mathrm{d} \phi \cos \left(k d_{a b} \cos \theta\right) \exp \left(-\frac{k_{\mathrm{B}} T}{2} k^{2} t^{2}\right. \\
& \times\left\{\frac{\left(\Delta_{Y}^{a} \cos \theta-\Delta_{Z}^{a} \sin \theta \sin \phi\right)^{2}}{J_{X}}+\frac{\left(\Delta_{X}^{a} \cos \theta-\Delta_{Z}^{a} \sin \theta \cos \phi\right)^{2}}{J_{Y}}\right. \\
& \left.\left.+\frac{\left(\Delta_{X}^{a} \sin \theta \sin \phi-\Delta_{Y}^{a} \sin \theta \cos \phi\right)^{2}}{J_{Z}}\right\}\right)+\mathcal{O}\left(\frac{t^{2}}{k^{2}}\right),
\end{aligned}
$$


where $\left(\Delta_{X}^{a}, \Delta_{Y}^{a}, \Delta_{Z}^{a}\right)$ designate the principal components of $\boldsymbol{\delta}_{i}^{a}$ and $d_{a b}=\left|\boldsymbol{\delta}_{i}^{a}-\boldsymbol{\delta}_{i}^{b}\right|$ are the distances between charges $a$ and $b$ within the same molecule.

At a given molecular geometry the two-dimensional integral (A3) can easily be calculated numerically. In the static limit $(t=0)$ this integral is taken analytically,

$$
g_{\mathrm{L}}^{(\mathrm{f})}(k)=\frac{1}{\mu^{2} k^{2}}\left(\sum_{a=1}^{M} q_{a}^{2}+\sum_{a \neq b}^{M} q_{a} q_{b} \frac{\sin \left(k d_{a b}\right)}{k d_{a b}}\right) .
$$

It is worth to remark that the formulas (A3), (A4) at sufficiently large values of wavevector $k$ can be applied to interacting systems as well, because then both intermolecular terms in (A1) and terms in (A3) caused by interactions (nonlinear in $t$ ) are small. 


\section{Figure captions}

Fig. 1. The longitudinal wavevector-dependent Kirkwood factor of the TIP4P water. The result obtained for the finite and infinite systems within the IS description using the ISRF geometry is plotted by dashed and solid curves, respectively. The corresponding result of the point dipole approximation for the infinite system is presented by open squares. The infinitesystem Kirkwood factor, evaluated in the Ewald and PDRF geometries, is shown as open circles and dotted curve, respectively. The long-short dashed curve corresponds to the Kirkwood factor in the free motion regime (equation (A4)).

Fig. 2. The longitudinal wavevector-dependent dielectric constant (a) of the TIP4P water within the IS description (circles connected by the solid curve) and the PD approximation (open squares). Two vertical lines indicate the positions of singularities. The function, describing the static screening of external charges in water, is shown in (b).

Fig. 3. The normalized, time autocorrelation functions of the longitudinal polarization fluctuations in the TIP4P water for the finite (circles) and infinite (solid curves) systems in the IS description as well as for the finite system in the PD approximation (dashed curves). The dotted curves show the self parts of the IS functions. The correlation functions of a noninteracting system, calculated using the exact relation (A2) and the short time approximation (A3), are shown by the long-short dashed curves and open circles, respectively. We note that five of the six dependencies are undistinguished in (h).

Fig. 4. The $3 \mathrm{D}$ plot of the wavevector- and time-dependent longitudinal polarization fluctuations in the IS description for the infinite system.

Fig. 5. The wavevector-dependent relaxation (circles) and correlation (squares) times for the longitudinal polarization fluctuations of the finite system. The results for the infinite systems are shown by the corresponding solid curves.

Fig. 6. The longitudinal wavevector- and frequency-dependent dielectric constant of the TIP4P water. The real and imaginary parts are shown as solid and dashed curves, respectively. Note the logarithmic scale in (a). Open squares connected by the long dashed curve in (b)-(e) reproduce the real part of the frequency-dependent dielectric constant at zero wavevector. The results obtained in the free motion regime (equation (A3)) are plotted in (f)-(h) as full (real part) and open (imaginary part) circles, respectively.

Fig. 7. The longitudinal wavevector- and frequency-dependent dielectric constant of the TIP4P water at great wavenumber values. Other features as for fig. 6 . 


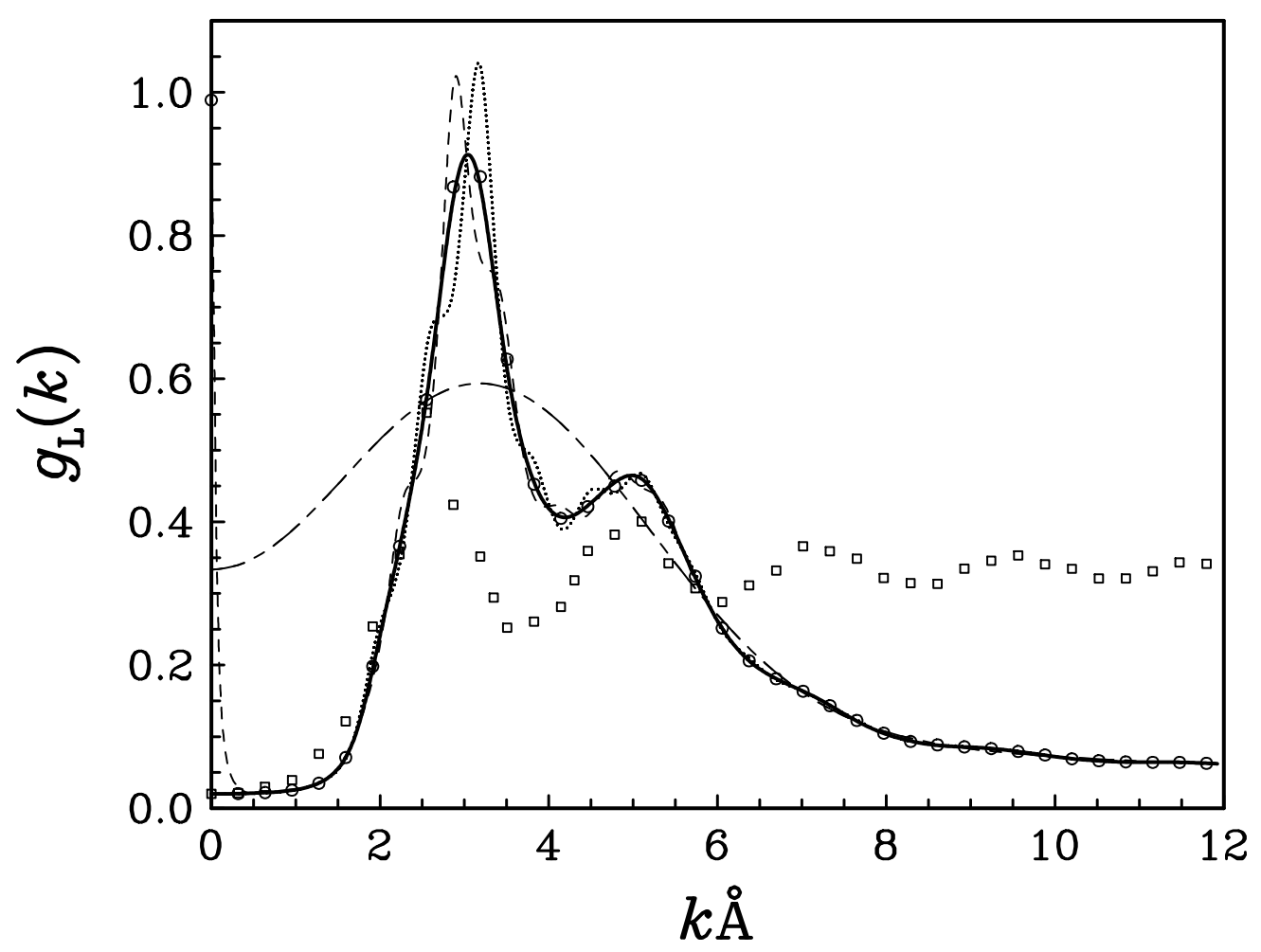

Fig. 1 

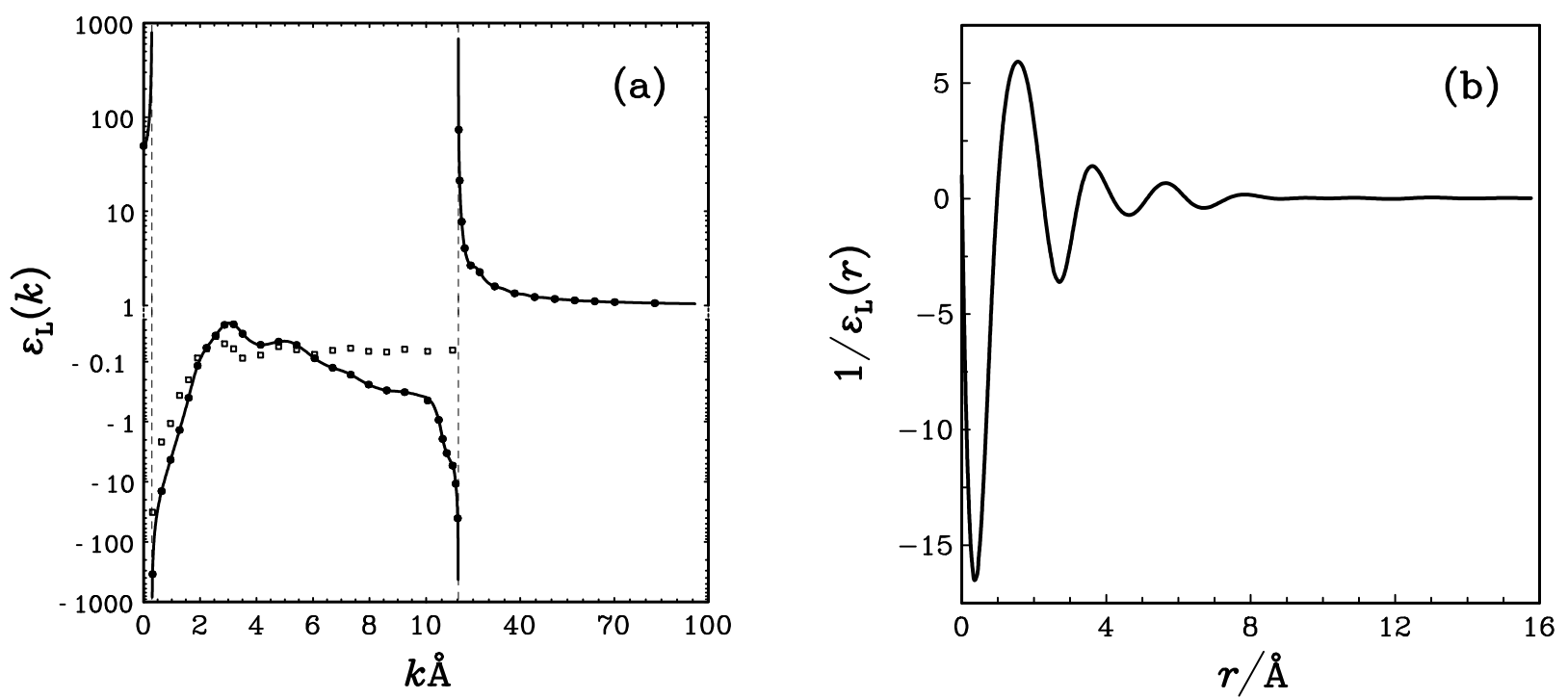

Fig. 2 

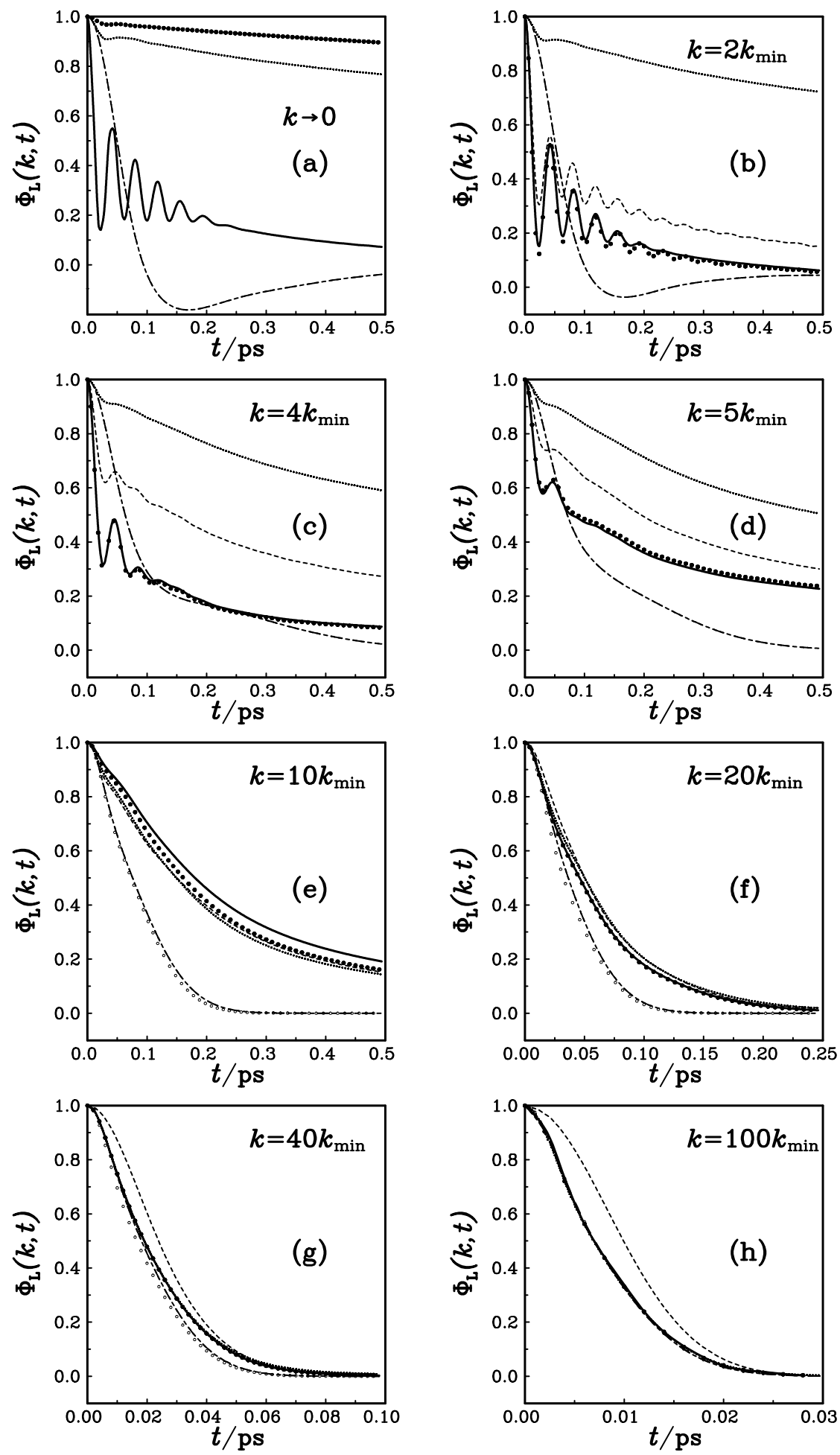

Fig. 3 


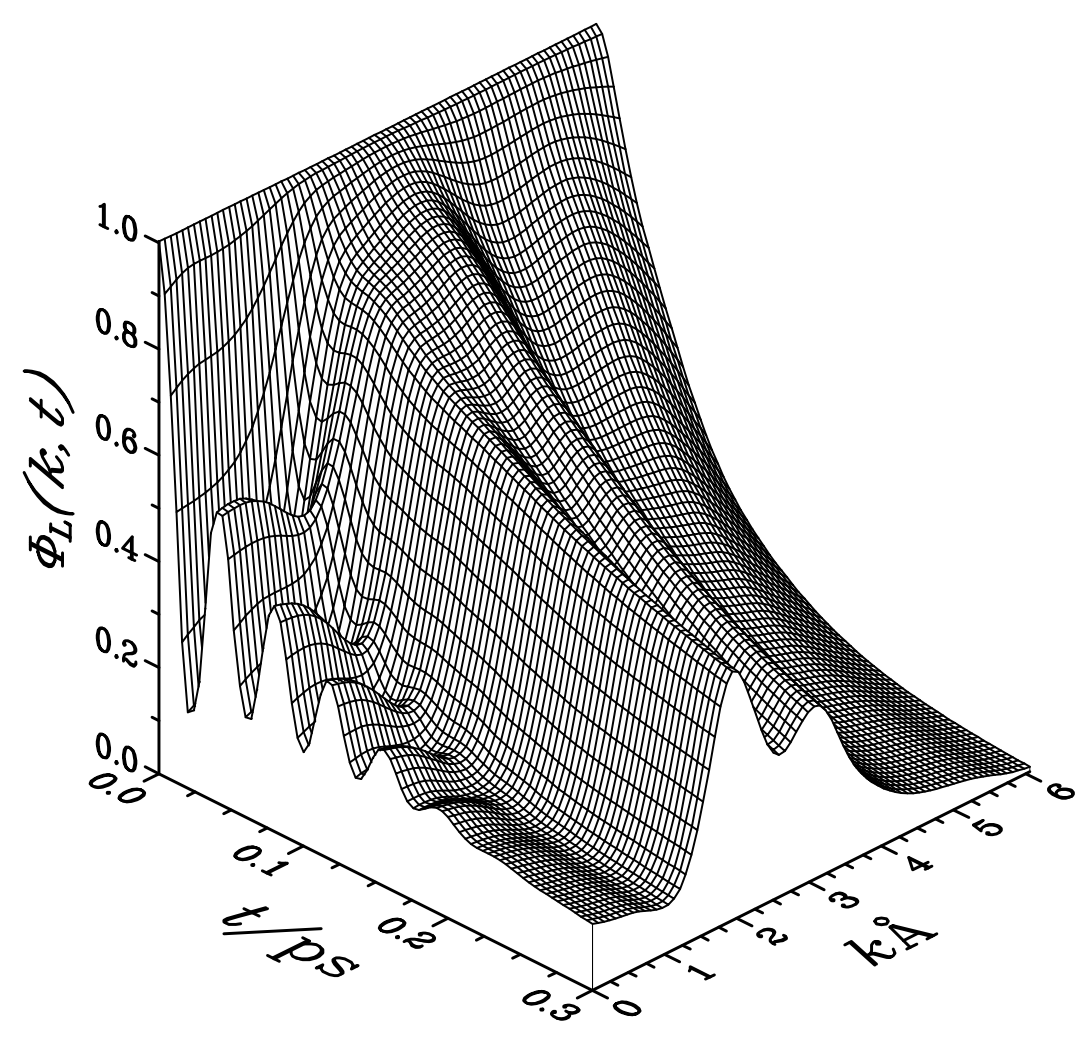

Fig. 4 


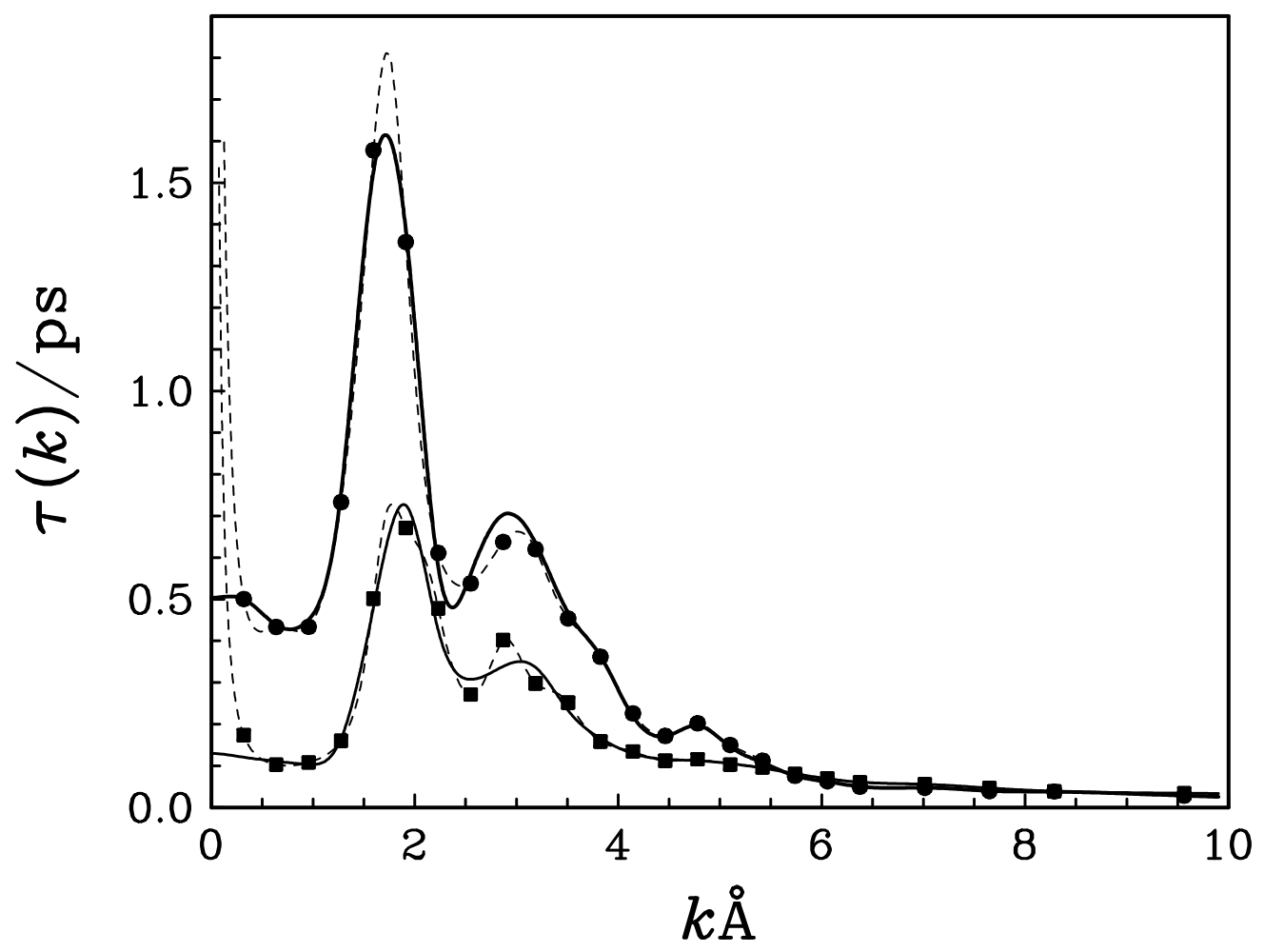

Fig. 5 

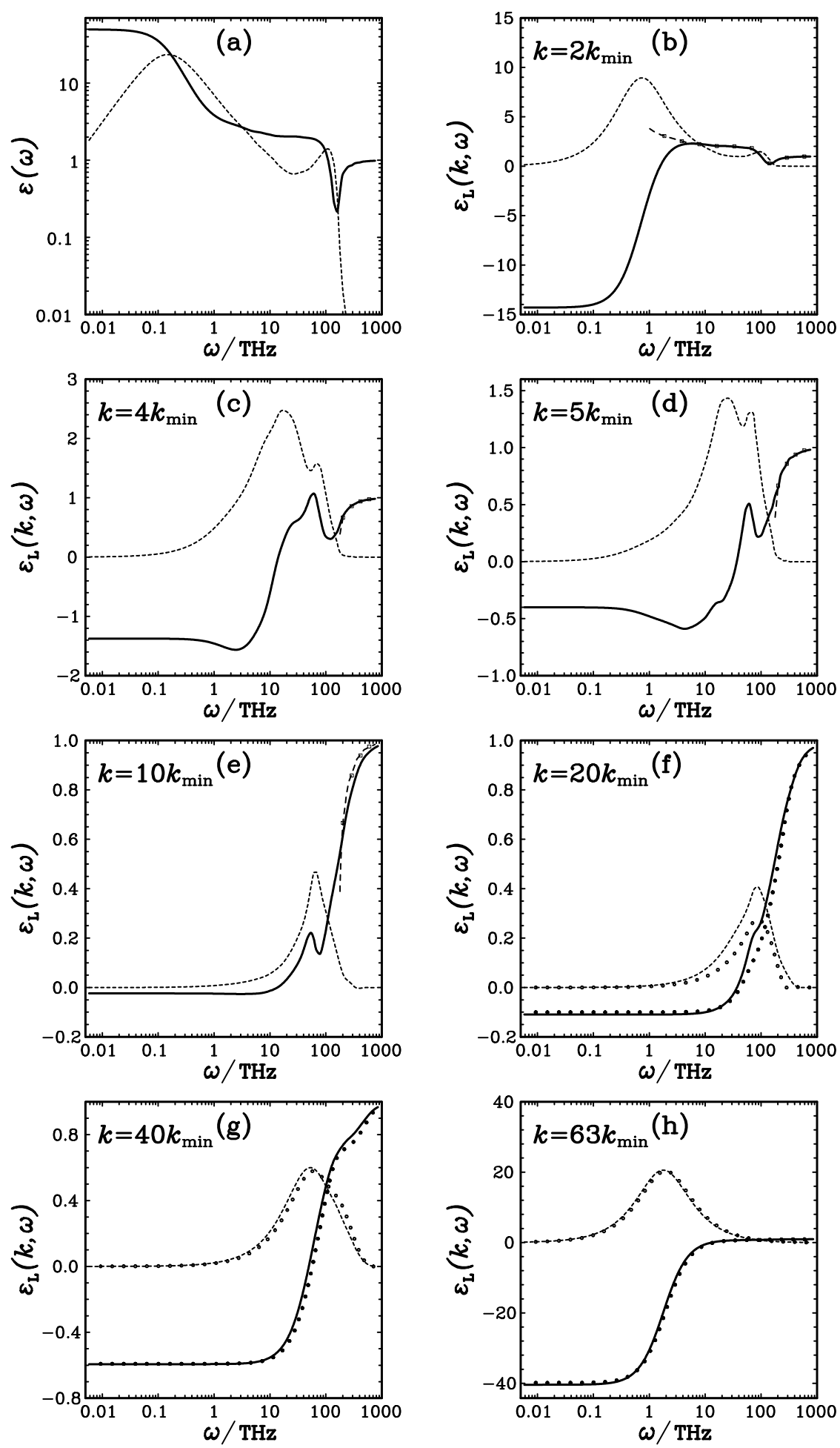

Fig. 6 

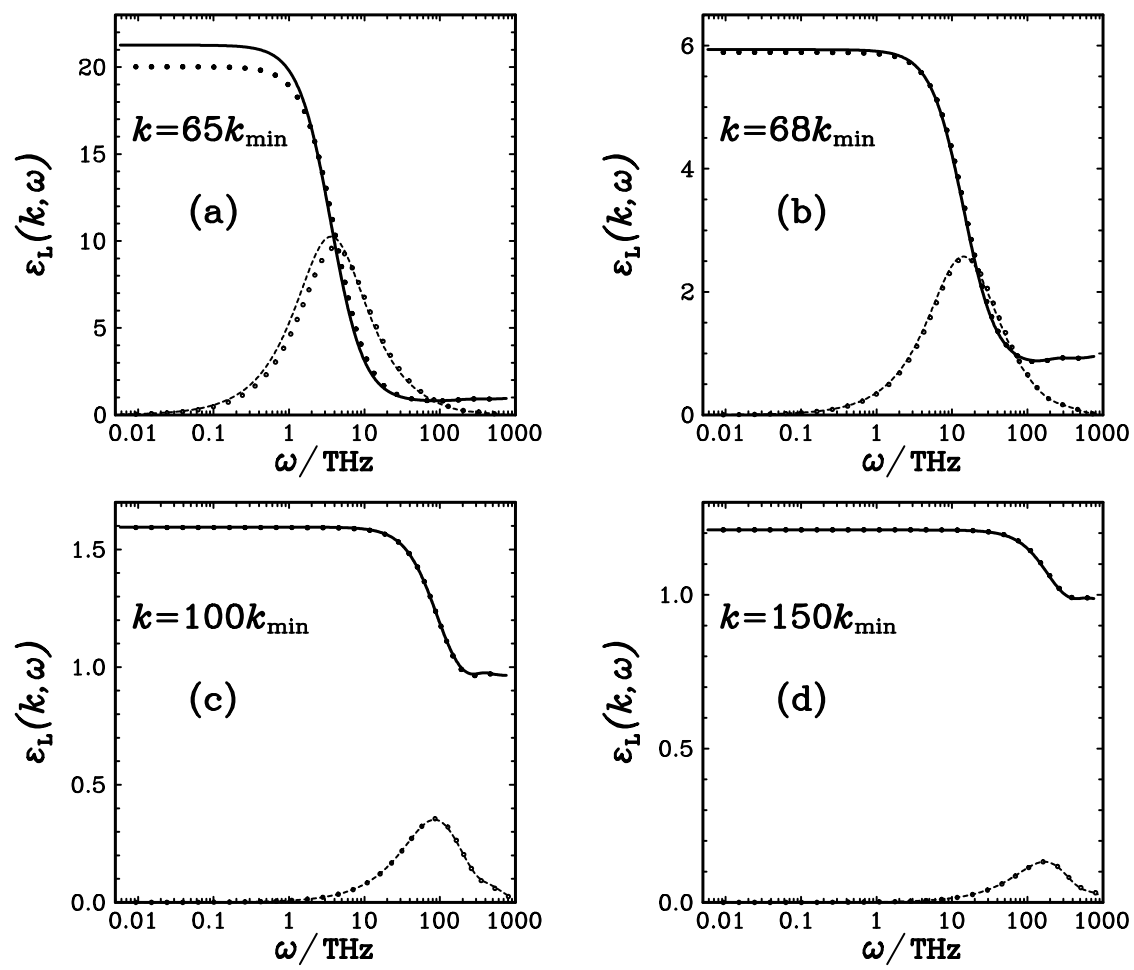

Fig. 7 